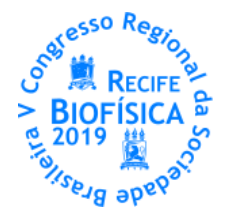

\title{
INTERAÇÃO DE AGREGADOS DE POPG NA PRESENÇA DE PEPTIDEO ANTIMICROBIANOS LL 37
}

\author{
Yeny Y. P. Valencia ${ }^{1}$, Gabriel C. A. da Hora ${ }^{1}$, Thereza A. Soares ${ }^{1}$, \\ 1'Departamento de Química Fundamental, Centro de Ciências Exatas e da Natureza, UFPE \\ *yaneth.uq@gmail.com
}

\begin{abstract}
INTRODUÇÃO
0 aumento da resistência aos antibióticos requer o desenvolvimento de novas formas de combater a infecção, um meio promissor para enfrentar esse desafio é representado pelos peptídeos antimicrobianos (AMPs) (ZASLOFF, 2002). Esses AMPs tem sido discutidos há muito tempo como uma possível nova classe de antibióticos que podem ser usados contra micróbios resistentes às drogas (WIMLEY; HRISTOVA, 2011). Ao contrário dos antibióticos convencionais, que interagem fortemente com moléculas alvo específicas, geralmente proteínas, a maioria dos AMPs age por um mecanismo inespecífico e frequentemente induz a morte celular a traves da ruptura da membrana plasmática. (WILDMAN; LEE; RAMAMOORTHY, 2003).
\end{abstract}

A ação na membrana plasmática pode seguir mecanismos similares de interação tais como barril, poro toroidal, agregados e carpete. (CATTE et al., 2018). O modelo barril (barril-stave) baseia-se em que os peptídeos são orientados perpendicularmente à superfície da membrana lipídica para formar poros. Também é possível que esses AMPs gerem poros toroidais, em que as bordas dos poros consistem de peptídeos e grupos de cabeça de lipídios que se dobram continuamente a partir da folha da bicamada superior até a bicamada inferior. Outro mecanismo de ação pode ser o modelo agregado, em que os peptídeos se ligam à membrana e conseguem atravessá-la formando complexos lipídeo - peptídeo sem obedecer nenhum padrão, más consegue atravessá-la totalmente. Finalmente o modelo carpete, começa por uma interação de tipo eletrostática entre o AMP e as cabeças dos fosfolipídios, em seguida o AMP se acumula na superfície de forma similar a um tapete, os peptídeos não atravessam a membrana, isto é, permanecem em posição horizontal à membrana. Após uma determinada concentração, causa destruição o qual gera a formação de micelas (CATTE et al., 2018).

0 peptídeo antimicrobiano LL37 é o primeiro peptídeo alfahelicoidal anfipático isolado de células humanas. Faz parte da primeira linha de defesa contra infecção local e invasão sistêmica de patógenos em locais de inflamação. Possui atividades antibacterianas e anti-biofilme e também é significativamente resistente à degradação proteolítica em solução. Apresenta uma carga de +6 . Essa carga é devido a que contém 6 resíduos de lisina e 5 de arginina contribuindo uma carga de +11 e 3 resíduos de glutamato e 2 de aspartato contribuindo uma carga de -5 fazendo um total de +6 (DÜRR; SUDHEENDRA; RAMAMOORTHY, 2006).

Dados preliminares por difração de raio-X de baixo ângulo (SAXS) sugerem que o LL37 desestabiliza bicamadas de POPG (1-Palmitoil2-Oleoil-sn-Glicero-3-[Fosfo-rac-(1-glicerol)]) e induz a formação de micelas com os peptídeos espacialmente localizados na superfície.
No presente projeto, pretendemos caracterizar o mecanismo molecular pelo qual o peptídeo antimicrobiano LL37 induz a desestabilização de agregados de POPG utilizando métodos de dinâmica molecular (MD) associados a modelos de tipo coarsegrained (CG). Membranas de POPG são comumente utilizadas como modelos experimentais representativos de células bacterianas, por tanto foram realizadas simulações computacionais para vários sistemas contendo POPG e LL37, onde foi avaliada a concentração do peptídeo e a influência da temperatura (Tabela 1).

\section{MATERIAIS E MÉTODOS}

Os sistemas simulados (tabela 1) foram solvatados com moléculas de água CG e adicionados $150 \mathrm{mM}$ de $\mathrm{NaCl}$ para reprodução da concentração fisiológica. O campo de força MARTINI (MARRINK et al., 2007; MARRINK; DE VRIES; MARK, 2004) foi usado em conjunto com o pacote de software GROMACS v4.6.7. (HESS et al., 2008)

O MARTINI é um campo de força para simulações de dinâmica molecular de biomoléculas Coarse - Grained (CG) parametrizado de forma sistemática. Na resolução CG, vários átomos são combinados em grupos funcionais conhecidos como super-átomos como se mostra na figura 1 , diminuindo o número de partículas no sistema e suavizando a paisagem energética o que permite passos de tempo de integração maiores e muitas vezes leva a uma dinâmica efetiva mais rápida em comparação as simulações com todos os átomos. (CARPENTER et al., 2017).
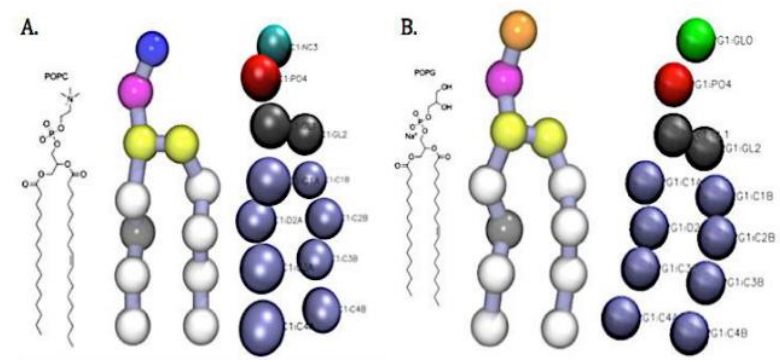

Figura 1. Representação esquemática das estruturas usadas neste trabalho. A. Modelo atomístico e coarse-grained do POPC. B. Modelo atomístico e coarse-grained do POPG. (Fonte: Coarse Grain Forcefield for Biomolecules, Martini).

As bicamadas de membrana CG foram construídas com o script INSANE (WASSENAAR et al., 2015) Os sistemas foram submetidas a uma otimização de energia e neutralizados através da adição de contra-íons $\mathrm{Na}+$ (apenas para POPG), seguido por uma segunda rodada de minimização de energia (5000 passos), equilibração de energia e dinâmica durante $3 \mu$ s ou $15 \mu$ sob condições NPT (temperatura e pressão constante). No caso dos sistemas de POPG com peptídeo LL37 foram necessários a adição de íons Cl-

(c) 2019 CC BY-NC-ND LICENSE. ANAIS: ENCONTRO ANUAL DA BIOFISICA (2019): 79-81. BIOFÍSICA E RADIOBIOLOGIA, BIOCIÊNCIAS, UFPE $21 \mathrm{E} 24$ DE MAIO, RECIFE, PERNAMBUCO, BRASIL ISSN: 2526-6071 ISSN: 2526-6071 
adicionais para neutralizar a carga total dos peptídeos. 0 termostato de $\mathrm{V}$-rescale foi usado para manter o sistema na temperatura de $300 \mathrm{~K}$. A pressão foi mantida em 1 bar através do acoplamento fraco das coordenadas das partículas e das dimensões da caixa no plano xy e ao longo do eixo $z$ por meio de escalonamento de coordenadas semi-isotrópicas com um tempo de relaxamento de 0,1 ps e uma compressibilidade de $4,5 \times 10^{-5}$ (kJ $\left.\mathrm{mol}^{-1} \mathrm{~nm}^{-3}\right)^{-1}$.Por fim, realizamos análises e discutimos propriedades estruturais derivadas desses simulações.

\begin{tabular}{|c|c|c|c|c|c|c|c|}
\hline System & & & & & $\begin{array}{c}\text { Temp. } \\
{[\mathrm{K}]}\end{array}$ & $\begin{array}{l}\text { Time } \\
\text { [us] }\end{array}$ & 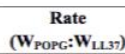 \\
\hline & Peptides & Lipids & Water & Ions & & & \\
\hline $\operatorname{cgPC}_{b}{ }^{300}$ & - & 520 & 5207 & 114 & 300 & 3 & - \\
\hline $\operatorname{cgPG}_{b}{ }^{300}$ & - & 520 & 4800 & 520 & 300 & 3 & - \\
\hline $\mathrm{cgPG}_{\mathrm{b}} 350$ & - & 520 & 4800 & 520 & 350 & 3 & - \\
\hline $\mathrm{cgPG}_{b}{ }^{400}$ & - & 520 & 4800 & 520 & 400 & 3 & - \\
\hline $\mathrm{cgPG}^{3 \mathrm{as}}$ & . & 600 & 6217 & 600 & 300 & 3 & - \\
\hline $\mathrm{cgPG}_{3 / 3}{ }^{300}$ & 6 & 520 & 13849 & 556 & 300 & 3 & 15:1 \\
\hline $\operatorname{cgPG}_{44^{3}}{ }^{300}$ & 8 & 520 & 13742 & 568 & 300 & 3 & 11:1 \\
\hline $\mathrm{cgPC}_{100}{ }^{300}$ & 10 & 520 & 8132 & 174 & 300 & 3 & $9: 1$ \\
\hline $\operatorname{cgPG}_{100}{ }^{300}$ & 10 & 520 & 9234 & 580 & 300 & 15 & 9:1 \\
\hline $\operatorname{cgPG}_{150}{ }^{300}$ & 15 & 520 & 22315 & 610 & 300 & 15 & 6:1 \\
\hline $\operatorname{cgPG}_{15 / 3} 300$ & 20 & 520 & 16858 & 640 & 300 & 15 & $4: 1$ \\
\hline $\mathrm{cgPG}_{15 / 3} 350$ & 20 & 520 & 16858 & 640 & 350 & 3 & 4:1 \\
\hline $\operatorname{cgPG}_{200} 300$ & 20 & 520 & 23558 & 640 & 300 & 3 & 4:1 \\
\hline $\mathrm{cgPG}_{200}{ }^{350}$ & 20 & 520 & 23558 & 640 & 350 & 3 & $4: 1$ \\
\hline $\mathrm{cgPG}^{2540,0} 300$ & 40 & 600 & 52877 & 840 & 300 & 3 & $3: 1$ \\
\hline $\mathrm{cgPG}_{400^{300}}$ & 40 & 612 & 19007 & 852 & 300 & 0.3 & 3:1 \\
\hline $\mathrm{cgPG}_{2020^{3}}^{300}$ & 40 & 2080 & 69954 & 2320 & 300 & 3 & 9:1 \\
\hline $\mathrm{cgPG}^{20220}{ }^{350}$ & 40 & 2080 & 69954 & 2320 & 350 & 3 & $9: 1$ \\
\hline $\mathrm{cgPG}^{20220200}$ & 40 & 2080 & 69954 & 2320 & 400 & 0.6 & 9:1 \\
\hline $\mathrm{cgPG}_{400}^{-300}$ & 40 & 2080 & 78394 & 2320 & 300 & 3 & 9:1 \\
\hline $\mathrm{cgPG}^{\circ}{ }_{\text {anisio }}^{300}$ & 40 & 2080 & 87835 & 2320 & 300 & 3 & 9:1 \\
\hline $\mathrm{cgPG}^{\circ}{ }^{300}$ & 60 & 2080 & 110289 & 2440 & 300 & 3 & $6: 1$ \\
\hline $\operatorname{cgPG}^{600}{ }^{6030}$ & 60 & 2080 & 110289 & 2440 & 350 & 0.3 & $6: 1$ \\
\hline 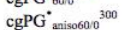 & 60 & 2080 & 119106 & 2440 & 300 & 0.3 & $6: 1$ \\
\hline $\mathrm{cgPG}_{\mathrm{y}}$ & . & 650 & 54550 & 650 & 300 & 3 & - \\
\hline
\end{tabular}

\section{RESULTADOS E DISCUSSÃO}

\section{POPG na interação com o LL37:}

Se obteve alguns observações interessantes, a primeira o peptídeo colocado imerso e em posição vertical com respeito à membrana foi puxado e orientado em posição paralela à superfície da bicamada 0 que era de esperar pelas fortes interações eletrostáticas do peptídeo positivamente carregado $(+6)$ e o lipídeo negativamente carregado (-1), e a segunda observação foi que o peptídeo não conseguiu atravessar a membrana como é mostrado nos perfis de densidade em numero para os primeiros 50 ns iniciais e finais mostrados na figura 2.

A.

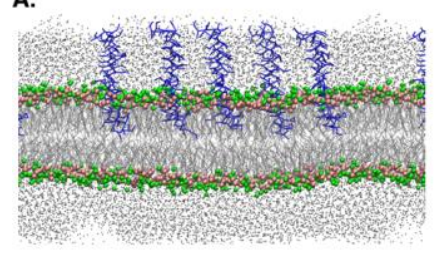

Parrial densitics
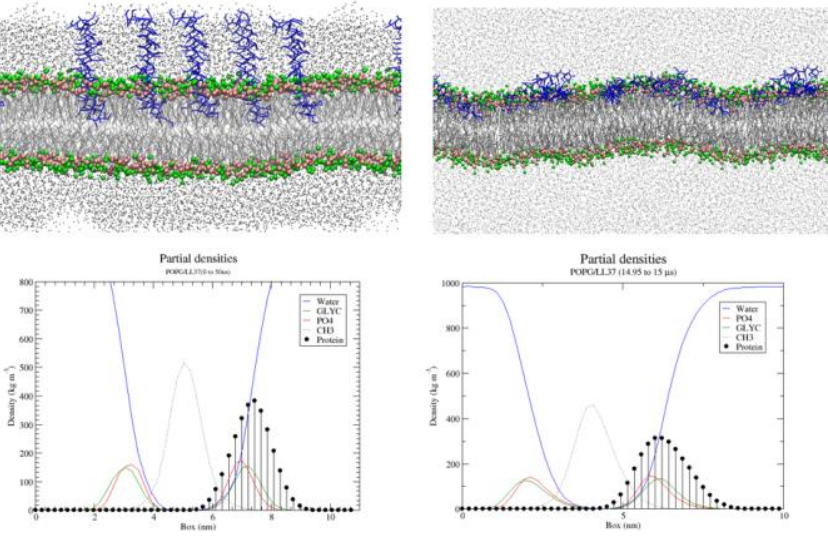

Figura 2.Representação da interação do peptídeo LL-37 com a bicamada fosfolipídica de POPG, $\operatorname{cgPG}_{300}{ }^{10 / 0}$ (acima) e seu perfil de densidade em numero (abaixo) para $\mathrm{A}$. primeiros 50 ns de simulação e B. 50 ns finais.

Estudo do efeito da concentração POPG - LL37:

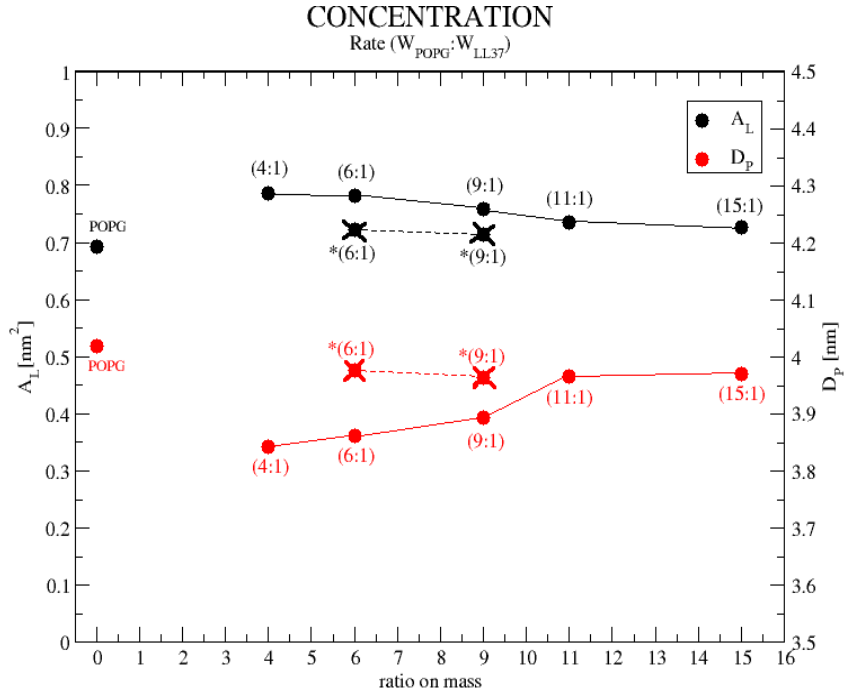

Figura 3. Avaliação da área por lipídeo $\left(A_{L}\right)$ e espessura $\left(D_{P}\right)$ em diferentes temperaturas para sistemas de 520 lipídeos $(\bullet)$ e sistemas gigantes com 2080 lipídeos $(\times)$

No gráfico 3, do lado esquerdo se encontra a área por cabeça e do lado direito a espessura e ambos estão em função da concentração, esses resultados são comparados com os resultados obtidos para POPG na ausência do peptídeo. Uma adição de poucos peptídeos na membrana de 520 lipídeos, é dizer a baixas concentrações de peptídeo (15:1 ou 11:1), causa um pequeno acréscimo na área por cabeça comparado com o POPG sem o peptídeo, e a medida que esta concentração aumenta a área por cabeça aumenta também. No caso da espessura uma pequena adição de peptídeos causa uma ligeira diminuição da espessura, quando chegamos a uma concentração de 9:1 observamos uma mudança evidente e a partir de esta a medida que aumentamos a concentração a espessura diminui rapidamente.

Para os sistemas gigantes com 2080 lipídeos o efeito é menor mas a tendência é a mesma. Um aumento na área por cabeça nos indica uma maior desordem nos lipídeos o que é refletido em uma ligeira inclinação dos grupos de cabeça, pelo que podemos afirmar que o LL-37 causa uma perturbação no sistema de bicamadas, por tanto uma curvatura.

\section{Estudo do efeito da temperatura:}

TEMPERATURE

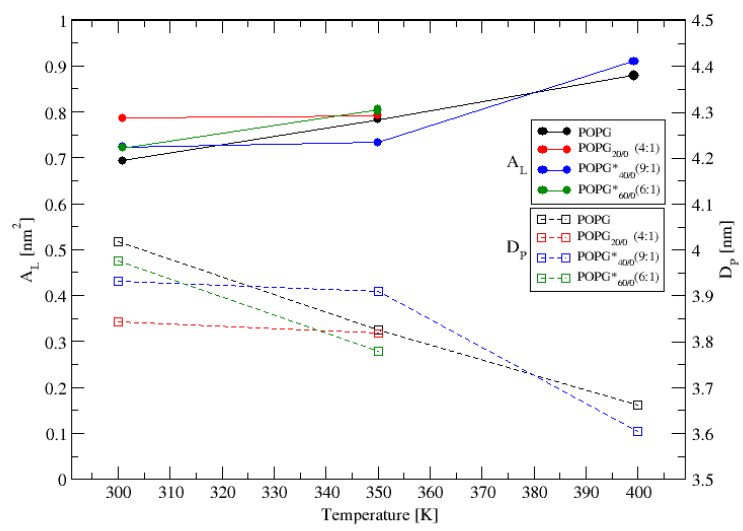

Figura 4. Avaliação da área por lipídeo (AL) e espessura (DP ) em diferentes temperaturas

Para POPG na ausência do peptídeo, observamos que a medida que a temperatura aumenta a área por lipídeo aumenta e a espessura diminui de forma lineal, para sistemas pequenos (vermelho) no 
existe mudanças significativas para uma concentração de 4:1, mas quando avaliamos sistemas maiores e em altas concentrações de peptídeo (azul e verde) observamos um incremento na área por lipídeo e uma diminuição na espessura.

\section{CONCLUSÔES}

Os resultados demonstram que o LL-37 se encontra na interface polar/ não polar da superfície da bicamada, essa orientação não muda o que rechaça a hipótese de um mecanismo de tipo barril.

Devido à falta de ter um peptídeo atravessando a membrana ao longo da simulação rechaçamos essa hipótese de um mecanismo de tipo poro toroidal. Concluimos que o LL-37 inicialmente forma um aglomerado e induz uma curvatura positiva seguido de uma desestabilição da membrana, via um mecanismo similar ao mecanismo carpete.

\section{REFERÊNCIAS}

WIMLEY, W. C.; HRISTOVA, K. Antimicrobial peptides: successes, challenges and unanswered questions. The Journal of membrane biology, Springer, v. 239, n. 1-2, p. 27-34, 2011.

ZASLOFF, M. Antimicrobial peptides of multicellular organisms. nature, Nature Publishing Group, v. 415, n. 6870, p. 389, 2002.

WILDMAN, K. A. H.; LEE, D.-K.; RAMAMOORTHY, A. Mechanism of lipid bilayer disruption by the human antimicrobial peptide, ll-37. Biochemistry, ACS Publications, v. 42, n. 21, p. 6545-6558, 2003.

CATTE, A. et al. Antimicrobial action of the cationic peptide, chrysophsin-3: a coarse-grained molecular dynamics study. Soft matter, Royal Society of Chemistry, v. 14, n. 15, p. 2796-2807, 2018.

DÜRR, U. H.; SUDHEENDRA, U.; RAMAMOORTHY, A. Ll-37, the only human member of the cathelicidin family of antimicrobial peptides. Biochimica et Biophysica Acta (BBA)-Biomembranes, Elsevier, v. 1758, n. 9, p. 1408-1425, 2006.

MARRINK, S. J.; DE VRIES, A. H.; MARK, A. E. Coarse Grained Model for Semiquantitative Lipid Simulations. The Journal of Physical Chemistry B, [s. l.], v. 108, n. 2, p. 750-760, 2004.

MARRINK, S. J. et al. The MARTINI Force Field: Coarse Grained Model for Biomolecular Simulations. The Journal of Physical Chemistry B, [s. l.], v. 111, n. 27, p. 7812-7824, 2007.

HESS, B. et al. GROMACS 4: Algorithms for Highly Efficient, LoadBalanced, and Scalable Molecular Simulation. Journal of Chemical Theory and Computation, [s. l.], v. 4, n. 3, p. 435-447, 2008.

CARPENTER, T. S. et al. Computational Lipidomics of the Neuronal Plasma Membrane. [s. l.], p. 2271-2280, 2017.

WASSENAAR, T. A. et al. Computational Lipidomics with insane : A Versatile Tool for Generating Custom Membranes for Molecular Simulations. Journal of Chemical Theory and Computation, [s. l.], v. 11, n. 5, p. 2144-2155, 2015.

MARTÍNEZ, L. et al. PACKMOL: A package for building initial configurations for molecular dynamics simulations. Journal of Computational Chemistry, [s. l.], v. 30, n. 13, p. 2157-2164, 2009. 